\title{
Mechanical Chest Compressions in an Avalanche Victim With Cardiac Arrest: An Option for Extreme Mountain Rescue Operations
}

\author{
Urs Pietsch, MD; Volker Lischke, MD; Christine Pietsch, MD; Karl-Heinz Kopp, MD \\ From the Kantonsspital St. Gallen, Institut für Anästhesiologie, St. Gallen, Switzerland (Dr U Pietsch); the Hochtaunus-Kliniken gGmbH, \\ Krankenhaus Bad Homburg, Abteilung für Anästhesie und Operative Intensivmedizin, Bad Homburg, Germany (Dr Lischke); the Schwarzwald-Baar- \\ Klinikum, Villingen-Schwennigen Klinik für Neurochirurgie, Villingen-Schwenningen, Germany (Dr C Pietsch); and the Air Zermatt Basis, Raron, \\ Switzerland (Dr Kopp).
}

\begin{abstract}
Mountain rescue operations often present helicopter emergency medical service crews with unique challenges. One of the most challenging problems is the prehospital care of cardiac arrest patients during evacuation and transport. In this paper we outline a case in which we successfully performed a cardiopulmonary resuscitation of an avalanche victim. A mechanical chest-compression device proved to be a good way of minimizing hands-off time and providing high-quality chest compressions while the patient was evacuated from the site of the accident.
\end{abstract}

Key words: accidental hypothermia, mechanical chest compression device, helicopter emergency medical service, out-of-hospital-resuscitation, avalanche, organ donation

\section{Case Report}

One early morning in April 2013, a group of 7 ski mountaineers was hit by an avalanche and buried in snow while ascending the Grünhornlücke (Switzerland). Six of the buried skiers freed themselves quickly, but 1 person could only be located and freed by his fellow mountaineers after a burial time of approximately 30 minutes. The mountaineers initiated basic life support (BLS) with cardiopulmonary resuscitation (CPR), as the victim did not show signs of life. Because of the remote location and the absence of mobile phone reception, the helicopter emergency medical service (HEMS) crew arrived on the scene approximately 60 minutes after the avalanche. The HEMS crew evaluated the patient and confirmed that he did not have a pulse. His pupils were dilated and unresponsive to light. After a short primary evaluation, the HEMS crew decided to continue with $\mathrm{CPR}$ and secure the airway by orotracheal intubation in accordance with the International Commission of Mountain Emergency Medicine (ICAR-MEDCOM) ${ }^{1}$ algorithm

Corresponding author: Urs Pietsch, MD, Kantonsspital St. Gallen, Institut für Anästhesiologie, Rorschacher Strasse 95, CH-9007 St. Gallen, Switzerland (e-mail: urs.pietsch@kssg.ch). as the burial time was less than 35 minutes and there was no snow in the patient's airway.

To deal with the fact that the technically difficult rescue from the steep incline and the transport into the hovering helicopter called for several longer episodes of hands-off time, it was decided within the first 2 minutes to establish a mechanical device to ensure continuous chest compressions. After a hands-off-time of approximately 45 seconds, the LUCAS 2 (Physio-Control, Inc, Lund, Sweden) chest compression system (Figure) was applied. The patient was then prepared for evacuation away from the avalanche site and approximately 12 minutes later loaded into the hovering helicopter. Once the chest-compression device was in place, continuous chest compressions and ventilation could be provided until the patient reached the hospital.

On admission into the hospital, the patient was in asystole, probably as a result of asphyxia, with dilated and nonresponsive pupils. An esophageal temperature of $24^{\circ} \mathrm{C}$ was measured. After establishing cardiopulmonary bypass, the patient was successfully warmed up and return of spontaneous circulation (ROSC) was obtained. Sadly, in the further clinical course, a brain scan revealed massive hypoxic brain damage in an otherwise uninjured patient, which implied that an asphyxic burial had taken 


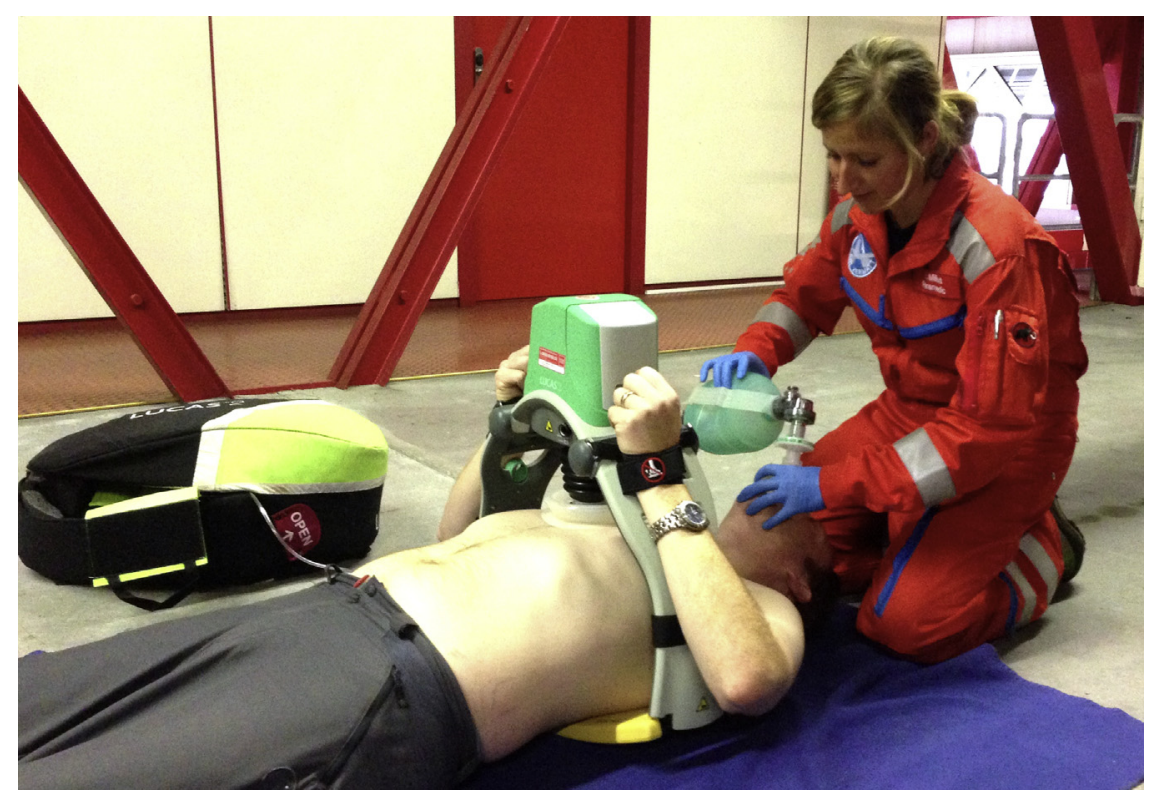

Figure. LUCAS 2 chest-compression device.

place under the avalanche or, alternatively, hypoxia could have occurred owing to insufficient BLS measures after the extrication After the patient was diagnosed as brain dead, the relatives consented to organ donation of the lungs, liver, and kidneys.

\section{Discussion}

\section{LESSONS LEARNED FOR PREHOSPITAL CARE IN CASES OF SEVERE HYPOTHERMIA}

This case demonstrates how demanding medical situations such as continuous CPR in mountain terrain require the HEMS crew to make quick decisions and provide solutions to complex cases. High-quality chest compression with minimal hands-off-time has been proven to be of critical importance for both the patient's survival rate and the neurological outcome after cardiac arrest. Even in relatively normal conditions of urban rescue, it can be difficult to control for these requirements, as several studies have shown. In difficult mountain terrain, if for example a patient has to be rescued from an avalanche in high altitude in a steep incline and then has to be transported or even winched up to the helicopter, the task of providing sufficient CPR becomes extremely difficult. Studies $^{2}$ have shown that interruptions of CPR caused by helicopter transport result in significantly reduced survival rates of these patients. Putzer et $\mathrm{al}^{3}$ observed that in simulated CPR in dummies during a helicopter transport the resuscitation provided by a mechanical device proved to be of higher quality and with less hands-off time than the one performed manually. So far, there are limited data ${ }^{3}$ in simulated scenarios suggesting that mechanical devices can be put to use to achieve good results in HEMS, but there has been no practical experience in a mountain setting. Our case report shows that mechanical devices can contribute to a significant increase in adequately performed CPR. We were able to show that a mechanical compression device was easy to apply and could minimize the hands-off time compared with the experience of previous similar cases in which manual CPR was performed.

The medical team should be alert that when using LUCAS 2, the position of the pressure pad should be controlled regularly and especially after changes in the patient's position to ensure an unchanged quality of chest compressions. A dislocation of the pressure pad can cause harmful injuries of intraabdominal organs. In contrast to LUCAS 2, AutoPulse is a band compression device. The correct placement of the band requires that the patient's upper body is lifted, thus asking for a hands-off time of approximately 20 seconds to place the base unit, position the Life Band, and start the compressions. Establishing the AutoPulse in steep or uneven surfaces could be more complicated compared with the LUCAS 2. However, the advantage of AutoPulse is that, once successfully placed, the patient is safely secured to the device and there is no risk of harmful malpositioning. The chest compressions are performed with a frequency of $80 / \mathrm{min}$ and a compression depth of 2 to $3 \mathrm{~cm}$. Although both compression rate and depth are not according to the latest guidelines, ${ }^{4,5}$ recent data have shown that the AutoPulse provides sufficient chest compressions. Given the direct influence of the quality of chest compression on the survival rate, ${ }^{6}$ it is evident 
Table. Staging of accidental hypothermia. The Swiss staging system $^{1,7}$

\begin{tabular}{llc}
\hline Stage & Clinical symptoms & Typical core temperature \\
\hline HT I & Conscious, shivering & $35^{\circ} \mathrm{C}-32^{\circ} \mathrm{C}$ \\
HT II & Impaired consciousness & $<32^{\circ} \mathrm{C}-28^{\circ} \mathrm{C}$ \\
HT III & Unconscious, vital signs & $<28^{\circ} \mathrm{C}-24^{\circ} \mathrm{C}$ \\
& present & \\
HT IV & No vital signs & $<24^{\circ} \mathrm{C}$ \\
\hline
\end{tabular}

HT = hypothermia.

that mechanical CPR provides a great benefit for patients suffering from hypothermia with a need of prolonged CPR until a spontaneous circulation can be reestablished.

Especially in patients with grade IV hypothermia (Table), the reduced body temperature can contribute to a reduced cerebral oxygen demand, so that even if the ROSC can be established only after hours of CPR, there is a chance of a good neurological outcome. ${ }^{7}$ Even in cases of poor neurological outcome and brain death of the patient, the use of mechanical chest-compression devices for potential organ donation can be a new opportunity in an attempt to increase the donor pool for organ transplantation. This can definitely be a reason for transport in spite of a grim prognosis such as our patient with suspected asphyxia as the case for the cardiac arrest. The duration of warm ischemia time from cessation of cardiac output until organ preservation is known as an important predictor of subsequent graft function. In controlled organ donation it is practical to limit warm ischemia times to less than 30 minutes. By contrast, in the situation of uncontrolled donors, as cardiac arrest has already occurred there is a much greater pressure on time to undertake the necessary ethical, legal, and practical procedures required to retrieve organs. Mechanical chest-compression devices have proved to be a valuable tool to maintain effective circulation after confirmation of cardiac death while the necessary steps are undertaken. ${ }^{8}$

With this knowledge in mind, it is difficult to decide when CPR in hypothermic patients should be abandoned. Unless an extreme hyperkalemia ( $>12 \mathrm{mmol} / \mathrm{L}$ ) is diagnosed, once the decision has been made to start CPR, the EMS should approach a center that can provide cardiopulmonary bypass, or better still, extracorporeal membrane oxygenation. ${ }^{7}$ Hospitals providing these advanced services are usually not in close proximity to mountain regions or accident sites, where most cases of hypothermia occur. Thus, until now there has always been the dilemma of a long-distance transport to a specialist hospital on the one hand and the abovementioned problems in performing sufficient CPR during helicopter transport on the other hand. Regarding these issues, mechanical chest-compression devices could theoretically improve the survival rate and neurological outcome in these patients; however, until today there are not enough data to make a clear recommendation. ${ }^{9}$

\section{Conclusions}

During a simulated cardiac arrest scenario in a helicopter rescue, the LUCAS $2^{3}$ has previously shown an increased CPR quality and reduced hands-off time, compared with manual chest compressions. Mechanical chest-compression devices could be of significant help to avoid longer hands-off time during complex mountain and flight rescue maneuvers, which is usually not possible if the compressions are performed manually. However, at this time there is insufficient evidence to recommend the routine use of mechanical chestcompression devices. The positive experiences made by single crews indicate a trend toward the beneficial effects of mechanical devices. Thus there is an urgent need for definitive clinical and cost-effectiveness trials to confirm or refute the routine use of mechanical chestcompression devices during resuscitation.

\section{References}

1. Brugger H, Durrer B, Elsensohn F, et al. Resuscitation of avalanche victims: evidence-based guidelines of the International Commission for Mountain Emergency Medicine (ICAR MEDCOM): intended for physicians and other advanced life support personnel. Resuscitation. 2013;84:539-546.

2. Thomas SH, Stone CK, Bryan-Berge D. The ability to perform closed chest compressions in helicopters. Am J Emerg Med. 1994;12:296-298.

3. Putzer G, Braun P, Zimmermann A, et al. LUCAS compared to manual cardiopulmonary resuscitation is more effective during helicopter rescue-a prospective, randomized, cross-over manikin study. Am J Emerg Med. 2013;31:384-389.

4. Soar J, Perkins GD, Abbas G, et al. European Resuscitation Council Guidelines for Resuscitation 2010 Section 8. Cardiac arrest in special circumstances: electrolyte abnormalities, poisoning, drowning, accidental hypothermia, hyperthermia, asthma, anaphylaxis, cardiac surgery, trauma, pregnancy, electrocution. Resuscitation. 2010;81:14001433.

5. Morrison LJ, Deakin CD, Morley PT, et al. Advanced Life Support Chapter Collaborators. Part 8: advanced life support: 2010 International Consensus on Cardiopulmonary Resuscitation and Emergency Cardiovascular Care Science With Treatment Recommendations. Circulation. 2010;122 (16 suppl 2):S345-S421.

6. Edelson DP, Abella BS, Kramer-Johansen J, et al. Effects of compression depth and pre-shock pauses predict 
defibrillation failure during cardiac arrest. Resuscitation. 2006;71:137-145.

7. Brown DJ, Brugger H, Boyd J, Paal P. Accidental hypothermia. N Engl J Med. 2012;367:1930-1938.

8. Morozumi J, Sakurai E, Matsuno N, et al. Successful kidney transplantation from donation after cardiac death using a load-distributing-band chest compression device during long warm ischemic time. Resuscitation. 2009;80: 278-280.

9. Paal P, Milani M, Brown D, Boyd J, Ellerton J. Termination of cardiopulmonary resuscitation in mountain rescue. High Alt Med Biol. 2012;13:200-208. 